\title{
Results from a Visual-Based Cervical Cancer Screening Program
}

\section{Yael Misrahi}

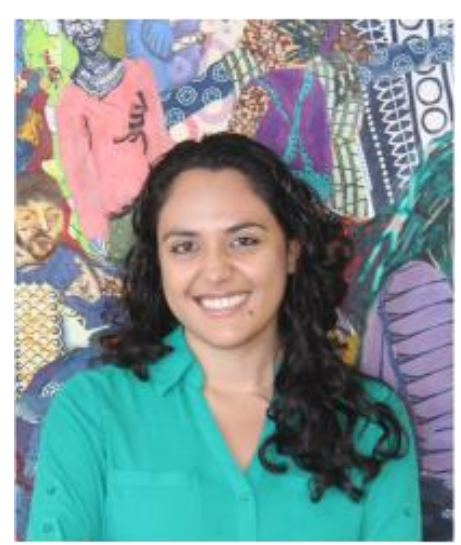

Editor's note: The 1st annual Future of Health: Telemedicine and Al summit was held on July 8, 2016 in Ho Chi Minh City, Vietnam. Ms. Misrahi was a member of the faculty, and the article that follows is based on her lecture.

Cervical cancer is the fourth most common cancer in women world-wide and the most common cancer in many low- and middle-income countries. 1 It is estimated that $84 \%$ of cervical cancer cases occur in less developed countries, where cervical cancer diagnostic screening and prevention methods are limited. To illustrate this disparity, Table 1 lists the countries with the top 20 highest incidence of cervical cancer. Compare these data to the United States, where the NIH (National Institutes of Health) reports an incidence rate for cervical cancer of approximately 8.1 cases per 100,000 women per year. $\underline{2}$

Over time, the incidence of cervical cancer in developed markets has been reduced thanks to the use of Pap smears (Pap test) and subsequent treatments, but women living in rural areas and low-resource settings (LRS) worldwide lack access to these relatively straightforward lifesaving techniques, and the demands of the test are too complex for many low-resource countries. Based on this background, the critical role for 
telemedicine as a platform for achieving quality controlled, accessible screening for cancer of the cervix becomes clear.

Table 1. Countries with the highest incidence of cervical cancer in 20121

\begin{tabular}{|lc|lc|} 
Country & $\begin{array}{c}\text { Age-Standardized } \\
\text { Rate/100,000 (World) }\end{array}$ & Country & $\begin{array}{c}\text { Age-Standardized } \\
\text { Rate/100,000 (World) }\end{array}$ \\
\hline Bolivia & 47.7 & Mali & 44.2 \\
\hline Burundi & 49.3 & Mozambique & 65.0 \\
\hline Comoros & 61.3 & Rwanda & 41.8 \\
\hline Fiji & 37.8 & Senegal & 41.4 \\
\hline Guinea & 38.4 & Suriname & 38.0 \\
\hline Guyana & 46.9 & Swaziland & 53.1 \\
\hline Kenya & 40.1 & Tanzania & 54.0 \\
\hline Lesotho & 38.4 & Uganda & 44.4 \\
\hline Madagascar & 44.6 & Zambia & 58.0 \\
\hline Malawi & 75.9 & Zimbabwe & 56.4 \\
\hline
\end{tabular}

\section{Visual Inspection with Acetic Acid (VIA) test}

One alternative screening approach is the visual Inspection with Acetic Acid (VIA) test, which enables the 'screen and treat' method recommended by the WHO (World Health Organization) guidelines, which ensures that a woman who screen positive is treated during the same visit as her screening. $\frac{3}{-}$

VIA is practiced by health providers in over 70 countries around the world, and has been studied and debated extensively as a high patient acceptance, low-cost screening alternative.

\section{Test Sensitivity}

In one systematic review and meta-analysis, the authors reported that regarding the HPV (human papillomavirus) test (cytology, cervical smear) versus unaided visual inspection with acetic acid (VIA) there was overtreatment with VIA (58 more per 1000 women). Differences in missed treatment ranged from 2 to 5 per 1000 women. For 1000 women screened positive and then sent to colposcopy, 464 would be falsely diagnosed with CIN grade 2-3 and treated. 
The authors concluded, "Although differences in sensitivity between tests could be interpreted as large, absolute differences in missed diagnoses were small. By contrast, small differences in specificity resulted in fairly large absolute differences in overtreatment." 4

\section{Patient Acceptance}

In clinical use, VIA is well accepted by patients. One study of women in Morocco revealed low awareness of cervical cancer (19.6\%) and a high acceptability of VIA screening (94.5\%). Among the 306 women screened, 99\% stated they would recommend the VIA testing to their friends and female relatives.

All women who were screened negative expressed their intent to repeat the test every three years. Those found VIA-positive affirmed they would perform confirmatory explorations. Almost all (96.3\%) of the women believed that screening by VIA could save their lives; cervical cancer was a concern for $98.6 \%$. There were $11.6 \%$ who felt anxious about repeating the VIA test. The majority of women (98.6\%) were satisfied with the service received at the health center. $\frac{5}{5}$

\section{Cost}

In a cost comparison of conventional cytology (Pap), VIA, and HPV DNA testing for detecting cases of cervical intraepithelial neoplasia grade 2+ (CIN2+) among HIVinfected women currently taking antiretroviral treatment at a public HIV clinic in Johannesburg, South Africa, it was reported that VIA was least costly.

Colposcopic biopsy costs largely drove the total and per case costs on average $\$ 67.71$ per procedure. In a higher volume scenario, the average cost per procedure was $\$ 3.67$ for VIA, \$8.17 for Pap and \$54.34 for HPV DNA.

VIA was least sensitive but most cost-effective at $\$ 17.05$ per true $\mathrm{CIN} 2+$ case detected. The cost per case detected for Pap testing was $\$ 130.63$ using a conventional definition 
for positive results and $\$ 187.52$ using a more conservative definition. HPV DNA testing was $\$ 320.09$ per case detected. $\underline{6}$

\section{Enhanced Visual Assessment (EVA)}

Ultimately, the success of any screening method requires convenient, cost-effective accessibility by a clinician who is qualified to perform the procedure and interpret the findings. Existing technology tends to be large and bulky, expensive, and not cloudconnected. It requires constant electricity supply and an expert colposcopist to be available at all times.

As a result, visual inspection of the cervix has emerged as a screening tool for LRS despite its limited specificity. This is because it provides immediate results, is accepted by patients, and is economical.

The Enhanced Visual Assessment (EVA) System (MobileODT) serves as a mechanism for telemedicine process improvement through the use of integrated imaging and a communications platform on inexpensive smartphones. It is compact and composed of three main elements: a handheld medical device (the Scope), a mobile phone-based application for documentation and provider communication (the App), and an online quality control and measurement and evaluation system (the Portal) (Figure 1). 


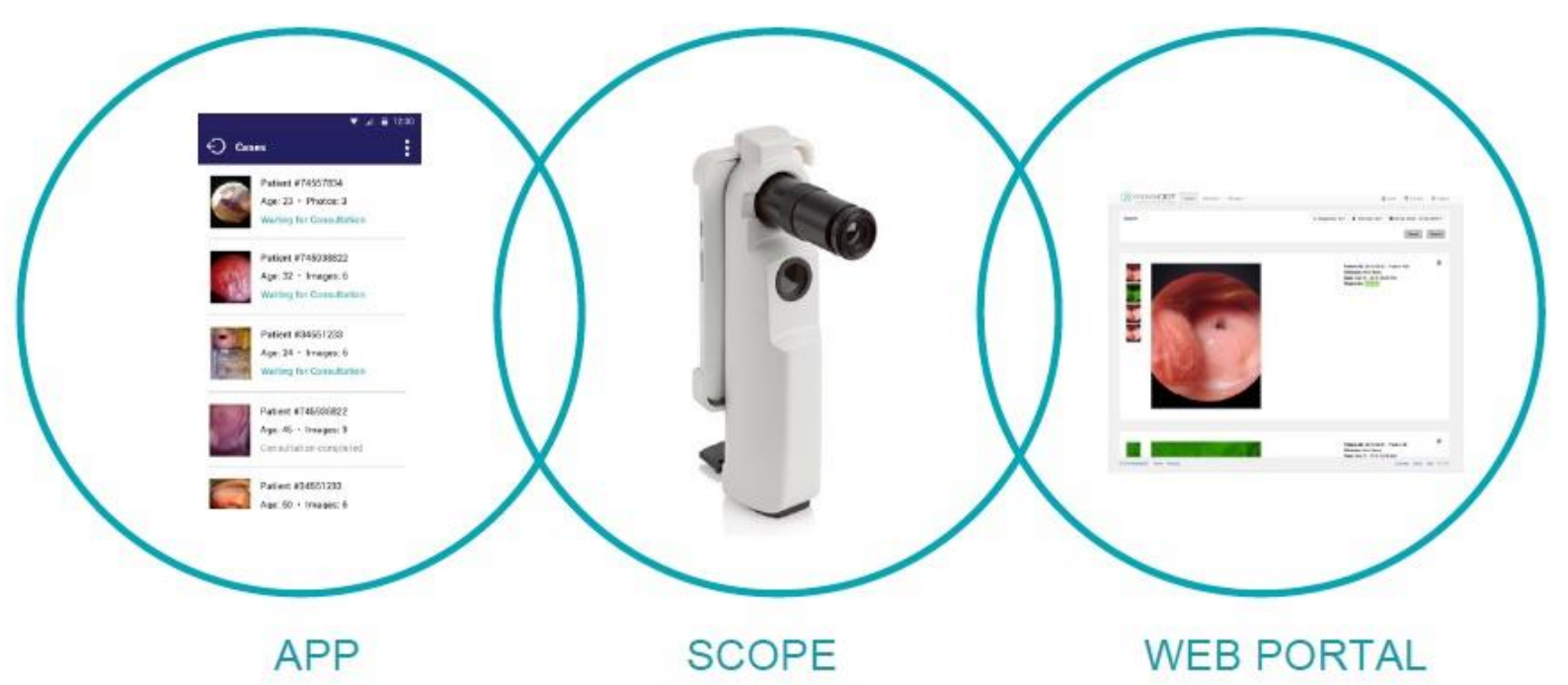

Figure 1. The EVA System handheld medical device (the Scope), with end-to-end system support for VIA/VILI (Lugol's lodine), post-HPV/Pap, and colposcopy

The EVA Scope, a mobile, lightweight, and durable medical device for photodocumentation and magnified inspection includes an ultra-bright light source with glarereducing cross-polarization technology, a 17x magnification lens, and a rechargeable battery for up to 10 hours of continuous examinations.

As a platform for telemedicine remote health, the EVA System functions on a mobile app, available on Android phones. The CervDx app enables providers using the EVA System to capture and review patient data confidentially, access remote expert consultations for decision support, participate in expert review and quality improvement opportunities, request consultation from an expert clinician in their network, initiate and track referrals, and engage and empower patients by providing education through image review.

Finally, all data and images captured on the CervDx app, are automatically stored and organized in the System's secure online web-portal. The EVA System is integrated through open APIs for sharing data with EMRs. 


\section{EVA in Clinical Practice}

In Kenya, as part of a cervical cancer screening camp, six organizations conducted cervical cancer screening using the EVA System. $\frac{7}{}$ These included the Health Ministry of Nairobi City County; Aga Kahn University Hospital; Family Health Options Kenya; Johns Hopkins International Program in Gynecology and Obstetrics, Kenya; SOS Children's Villages, Kenya; and Population Services, Kenya.

As part of the program, nurses conducting the visual screening were first trained to use the EVA System, including the app. Training was conducted the morning that the screening camp began. It emphasized the standard VIA workflow and how EVA can be used to augment the procedure. Trainers remained onsite throughout the camp to help troubleshoot any technical issues and provide ongoing guidance. A MobileODT video of the training process can be viewed here.

During screening, routine VIA procedures were followed. A speculum was inserted into the vagina to open the vaginal canal and enable visualization of the cervix. A thin layer of acetic acid was applied using an elongated swab, which removes moisture from the cells and turns areas with cervical dysplasia white over approximately 2 minutes (the acetowhitening process is also used in colposcopy procedures in higher level clinics).

The cervix was illuminated from outside the patient's body and visualized by the clinician. If the clinician observed white area(s) on the cervix, the test result was considered positive. An example image following the procedure is shown in Figure 2. 


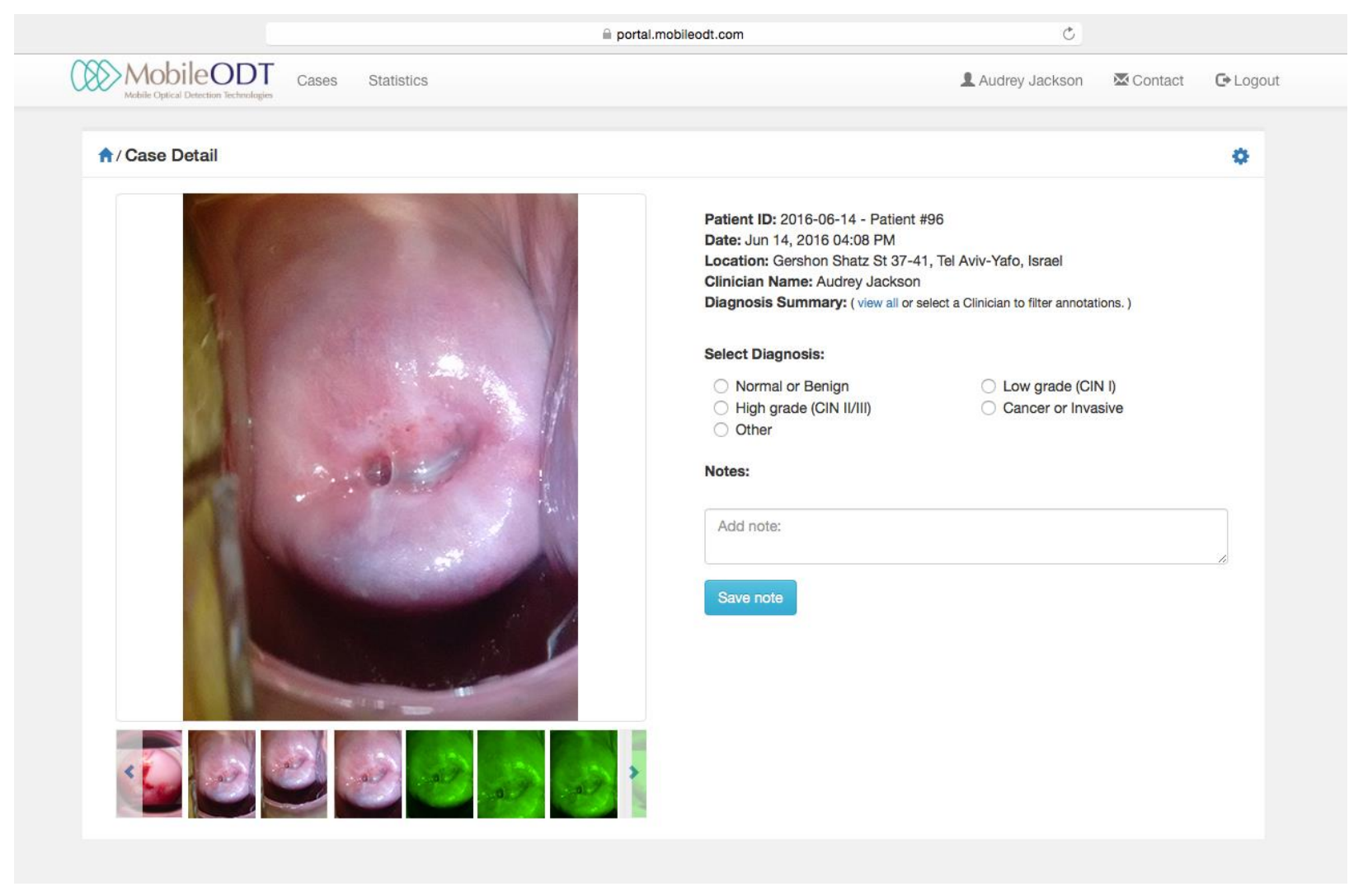

Figure 2. Sample visualization of the cervix using the EVA System

Aggregated screening and treatment data from the screening camp revealed that 824 women were screen with enhanced VIA/VILI (Lugol's solution), and 85 (10.3\%) were treated with cryotherapy. Among the women screened, $86.7 \%$ showed no signs of cancer or precancerous lesions, while $12.6 \%$ had precancerous lesions, with $0.7 \%$ had suspected cancer (Figure 3) 


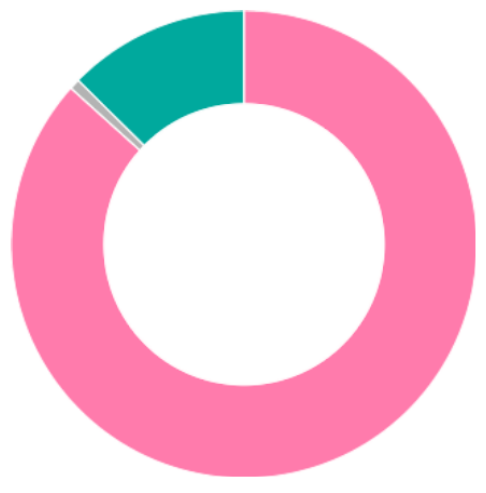

OF 824

WOMEN SCREENED:

- $86.7 \%$ NO SIGNS OF CANCER OR PRECANCEROUS LESIONS

- $12.6 \%$ PRECANCEROUS LESIONS

- $0.7 \%$ SUSPECTED CANCER

Figure 3. Aggregated screening and treatment data collected as part of a cervical cancer screening camp in which six organizations conducted cervical cancer screening using the EVA System ( $\mathrm{N}=824)$

\section{Discussion}

Cervical cancer is a leading cause of death among women in emerging markets. Yet, mortality can be minimized. As a slow to develop disease, whose visual markers can be identified years before the patient is in danger, cervical cancer is also very treatable. When pre-cancerous growths are diagnosed in their first five years, treatment can be applied in virtually any setting for less than \$28USD.

The key to early treatment is early diagnosis, and a critical component of early diagnosis across all clinical approaches is the visual inspection of the cervix to determine the type, location, and extent of disease. In rural and LRS settings, technology such as the EVA System can play a key role in implementing cost effective screening.

Yael Misrahi leads the business development efforts of MobileODT, with a specific focus on developing partnerships with international non-profits and global health organizations. 
Tags: 1st annual Future of Health: Telemedicine and Al summit, CervDx, cervical cancer, cervix, colposcopic biopsy, how does telehealth enhance the doctor patient relationship, HPV, human papillomavirus, Kenya, low-resource settings, LRS, mobile app, MobileODT, screening, telehealth, telemedicine, telemedicine process improvement, telemedicine program implementation, telemedicine remote health, VIA, visual inspection with acetic acid, what is telemedicine and how does it work, Yael Misrahi

\section{References}

1. World Cancer Research Fund International. Cervical cancer statistics. 2015. URL: http://www.wcrf.org/int/cancer-facts-figures/data-specific-cancers/cervicalcancer-statistics. Accessed 8/16/16.

2. USDHHS. Cervical cancer. 2013. https://report.nih.gov/nihfactsheets/viewfactsheet.aspx?csid=76. Accessed 8/16/16.

3. WHO. WHO guidelines for screening and treatment of precancerous lesions for cervical cancer prevention. 2013. URL:

http://apps.who.int/iris/bitstream/10665/94830/1/9789241548694 eng.pdf. Accessed 8/16/16.

4. Mustafa RA, Santesso N, Khatib R, et al. Systematic reviews and meta-analyses of the accuracy of HPV tests, visual inspection with acetic acid, cytology, and colposcopy. Int J Gynaecol Obstet. 2016 Mar;132(3):259-65. URL: http://www.ijgo.org/article/S0020-7292(15)00663-3/pdf. Accessed 8/16/16.

5. Selmouni F, Zidouh A, Alvarez-Plaza C, El Rhazi K. Perception and satisfaction of cervical cancer screening by Visual Inspection with Acetic acid (VIA) at Meknes-Tafilalet Region, Morocco: a population-based cross-sectional study. BMC Womens Health. 2015 Nov 24;15:106.

https://bmcwomenshealth.biomedcentral.com/articles/10.1186/s12905-015-0268… Accessed 8/16/16.

6. Lince-Deroche N, Phiri J, Michelow P, Smith JS, Firnhaber C. Costs and cost effectiveness of three approaches for cervical cancer screening among HIV- 
positive women in Johannesburg, South Africa. PLoS One. 2015 Nov 16;10(11):e0141969. URL:

http://journals.plos.org/plosone/article?id=10.1371/journal.pone.0141969. Accessed 8/16/16.

7. Peterson CW, Rose D, Mink J, Levitz D. Real-time monitoring and evaluation of a visual-based cervical cancer screening program using a decision support job aid. Diagnostics (Basel). 2016 May 16;6(2). URL: http://www.mdpi.com/20754418/6/2/20/pdf. Accessed 8/16/16.

8. Quentin W, Adu-Sarkodie Y, Terris-Prestholt F, et al. Costs of cervical cancer screening and treatment using visual inspection with acetic acid (VIA) and cryotherapy in Ghana: the importance of scale. Trop Med Int Health. 2011 March; 16(3): 379-389. URL: https://www.ncbi.nlm.nih.gov/pmc/articles/PMC3429861/. Accessed 8/16/16. 